
\title{
Application of Superconducting Hot-Electron Bolometer Mixers for Terahertz-Band Astronomy
}

Hiroyuki MAEZAWA ${ }^{\dagger a)}$, Nonmember

\begin{abstract}
SUMMARY Recently, a next-generation heterodyne mixer detectora hot electron bolometer (HEB) mixer employing a superconducting microbridge- has gradually opened up terahertz-band astronomy. The surrounding state-of-the-art technologies including fabrication processes, 4 $\mathrm{K}$ cryostats, cryogenic low-noise amplifiers, local oscillator sources, micromachining techniques, and spectrometers, as well as the HEB mixers, have played a valuable role in the development of super-low-noise heterodyne spectroscopy systems for the terahertz band. The current developmental status of terahertz-band HEB mixer receivers and their applications for spectroscopy and astronomy with ground-based, airborne, and satellite telescopes are presented.

key words: superconducting hot-electron bolometer mixer receiver, astrophysics, heterodyne spectroscopy, terahertz
\end{abstract}

\section{Introduction}

Many spectral lines for rotational, rotation-vibration, and fine-structure transitions of gas species in the interstellar medium lie in the millimeter to submillimeter wave band. In this frequency band heterodyne spectroscopy with high-frequency resolution is a powerful tool for identifying the line-of-sight velocity components of interstellar media such as dense molecular clouds, the warm and cold neutral medium, and the warm ionized medium, as shown in Fig. 1. Such identifications promote an accurate understanding of their basic physical conditions (dynamics, densities, and temperatures), chemical conditions, and formation and evolutionary processes of star-forming regions, their parent interstellar clouds, and galaxies. The $2.45-$ and $1.47-\mathrm{THz}$ band fine-structure transition $\left({ }^{3} P_{2}-{ }^{3} P_{1}\right.$ and $\left.{ }^{3} P_{1}-{ }^{3} P_{0}\right)$ lines of [N II] are important tracers of the extended warm and low density ionized layer and localized HII regions in the galaxy. The 4.7- and 2.06- THz $\left({ }^{3} P_{1}-{ }^{3} P_{2}\right.$ and $\left.{ }^{3} P_{0}-{ }^{3} P_{1}\right)$ transition lines of $[\mathrm{OI}]$ act as important coolants of neutral clouds under high UV fields and/or high densities $\left(>10^{3.5} \mathrm{~cm}^{-3}\right)$ in our galaxy, whereas the $1.9-\mathrm{THz}^{2} P_{3 / 2}-{ }^{2} P_{1 / 2}$ transition line of [C II] is important for cooling neutral clouds under lower densities and weak UV fields [1], [2]. These cooling processes play a critical role in the gravitational collapse of dense cores resulting in star formation. [CII] and [N II] also play a key role in producing the basic molecules such as ionized molecules, hydrocarbons, nitrogen hydrides, and oxide

Manuscript received June 30, 2014.

Manuscript revised October 22, 2014.

${ }^{\dagger}$ The author is with the Department of Physical Science, Osaka Prefecture University, 1-1 Gakuen-cho, Naka-ku, Sakai, Osaka 599-8531, Japan.

a)E-mail: maezawa@p.s.osakafu-u.ac.jp

DOI: 10.1587/transele.E98.C.196

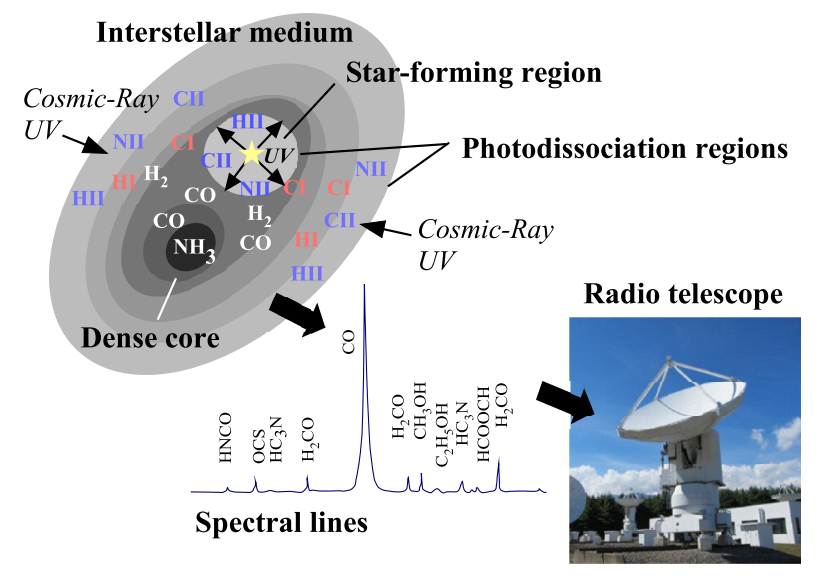

Fig. 1 Schematic illustration of heterodyne spectroscopy in astronomy.

species, which subsequently form various complex species including organic molecules in dark clouds. The highly excited dense gaseous species heated in star-forming regions have many lines in the terahertz frequency bands.

Molecular species in earth's atmosphere and other planetary atmospheres emit spectral lines efficiently in the radio to infrared bands. The profiles of these lines are shaped by superimpositions of emission from molecules at each altitude. In case of the earth, molecules excited thermally in the upper atmosphere have Gaussian-type line profiles with a Doppler width prevailing, whereas those in the lower atmosphere exhibit Lorentzian profiles because of pressure broadening. High spectrally resolved and wideband heterodyne sensing make it possible to retrieve the spectral lines of atmospheric minor constituents related to global warming and ozone-recovering/destruction processes (e.g., $\mathrm{H}_{2} \mathrm{O}, \mathrm{O}_{3}, \mathrm{NO}_{x}$, hydro radicals, and sulfurand chlorine-containing species). Various key spectral lines from such gaseous species also lie in the $\mathrm{THz}$ band.

Despite its scientific and observational importance, 1$10 \mathrm{THz}$ band radio astronomy has been long unexplored because of a lack of good observing sites and the unavailability of highly sensitive heterodyne receivers in this frequency range. Niobium-based superconductor-insulatorsuperconductor (SIS) mixers have allowed remarkable development of millimeter to submillimeter wave band astronomy. In a niobium-based SIS junction, a thin layer of aluminum oxide is a very good match with the niobium $(\mathrm{Nb})$ electrode, which gives an excellent insulator with high critical density and very low leakage current. Because of the 
resultant strong nonlinearity, the SIS junction works as a super-low-noise mixer detector. Above the gap frequency of $\mathrm{Nb}, f_{\text {gap }}=\Delta / h=\sim 670 \mathrm{GHz}$, where $\Delta$ and $h$ are the superconducting energy gap and Planck's constant, respectively, the electrodes and integrated tuning circuit become lossy owing to the breaking of Cooper pairs [3]. To avoid the loss, a low-loss normal conductor such as aluminum and a superconductor film such as niobium titanium nitride (NbTiN) with a high $f_{\text {gap }}$ are widely utilized. Recently, the band 10 receiver of Atacama Large Millimeter/Submillimeter Array (ALMA) project for $780-950 \mathrm{GHz}$ frequency band has achieved a quantum-noise-level equivalent noise temperature of $250 \mathrm{~K}$ (double side band (DSB)), corresponding to $5 h v / k_{B}$ [4], where $k_{B}$ is the Boltzmann constant. Above twice the energy gap frequency of $\mathrm{Nb}(\sim 1.3 \mathrm{THz}) \mathrm{Nb}$-based SIS junctions cannot function as a detector, at least in principle. Against this background, the superconducting hotelectron bolometer (HEB) mixer is being developed as the next-generation of heterodyne mixer for operation above 1 THz.

\section{Heterodyne Receiver}

Radio-frequency (RF) signals above $0.1 \mathrm{THz}$ are difficult to amplify directly with high frequency response and high sensitivity. Therefore, the input RF signal is first mixed with a local oscillator (LO) signal using a super-low-noise mixer detector cooled with a $4 \mathrm{~K}$ cryocooler, and subsequently the down-converted intermediate frequency (IF) signal is amplified by cooled low-noise amplifiers for $\mathrm{L}(0.5-1.5 \mathrm{GHz}), \mathrm{S}$ (2-4 GHz), C (4-8 GHz), or X (4-12 GHz) band as shown in Fig. 2. The RF signal is led to the mixer via a low-loss vacuum window (e.g., of high-density polyethylene) and an infrared filter (e.g., Zitex 106) for suppression of thermal radiation. Additionally, for an HEB mixer a cooled band eliminator such as a mesh filter is inserted for suppression of the direct detection effect [5]-[7]. In several terahertz band, at present the most well-used LO sources are IRpumped gas lasers and continuous-wave (CW) solid-state oscillators consisting of amplifier-multiplier chains coupled with a commercial high-quality microwave signal generator (SG). At $2.7 \mathrm{THz}$ the latter output power has achieved $\sim 3 \mu \mathrm{W}$ [8], which is sufficient to fully pump an HEB mixer. The frequency and/or phase of the primary microwave signal output from the SG is precisely locked with the highlystabilized reference signal from a GPS or hydrogen maser atomic clock.

Finally, via adequate amplifications the IF signal is spectrally resolved with spectrometers. Recently, highresolution, wideband digital fast Fourier transfer spectrometers (FFTSs) employing gigahertz analog-to-digital converters and field programmable gate array chips have become commercially available. For example, the RPG extended bandwidth XFTTS has an instantaneous bandwidth of 2.5 $\mathrm{GHz}$ and 32768 frequency channels [9]. The Agilent Acqiris AC240 has a 1-GHz bandwidth and 16384 channels. These heterodyne spectroscopy units provide us with a fre-

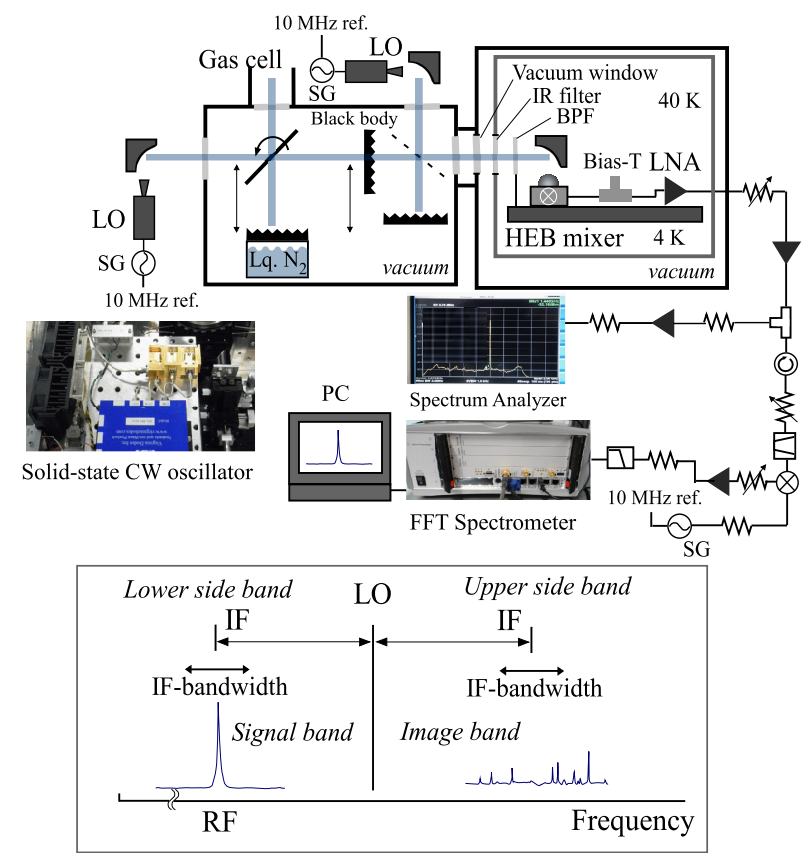

Fig. 2 Schematic drawings of heterodyne detection and a receiver system for heterodyne spectroscopy.

quency resolution of $<100 \mathrm{kHz}$, making them powerful tools for precisely resolving the narrow spectral lines of gaseous species exited thermally at low pressure.

The observable spectral coverage of the velocities of astronomical target, $\Delta V$, is determined by the IF bandwidth of the heterodyne system as explained by $\Delta V=\mathrm{c} \times \Delta B / f_{R F}$, where $\Delta B$ is the bandwidth of the spectrometer, $f_{R F}$ is the frequency of the RF signal, and $\mathrm{c}$ is the velocity of light. Generally spectral lines come from a number of interstellar clouds with different velocity components lying along the line of sight. The typical total width of the spectral lines observed toward the galactic disk is less that $100 \mathrm{kms}^{-1}$, while the line width of some kind of interstellar gaseous species in the galactic center reaches $\sim 600 \mathrm{~km} \mathrm{~s}^{-1}$ which corresponds to the instantaneous IF bandwidth of $\sim 4 \mathrm{GHz}$, for example, for $2 \mathrm{THz}$ band observations.

Largely, the RF bandwidth of THz-band HEB mixer receivers is limited by impedance matching of the input radiation and of the quasi-optical antenna or waveguide-type feed or by the output frequency range of local sources, while the instantaneous IF bandwidth is limited by the 3-dB roll-off IF bandwidth (of several gigahertz) of the HEB mixer. For SIS mixer receivers the IF bandwidth is usually restricted by that of the spectrometer or of the low-noise cryogenic amplifier and isolator. By using parallel-connected spectrometers instantaneous spectroscopic bandwidth can be practically broadened.

In heterodyne detection, the system noise temperature is expressed as

$$
\begin{aligned}
T_{S Y S} & =T_{R X} \times e^{\tau}+T_{S K Y}\left(e^{\tau}-1\right), \\
T_{R X} & =T_{R F}+\frac{T_{M I X}}{G_{R F}}+\frac{T_{I F}}{G_{R F} G_{M I X}},
\end{aligned}
$$



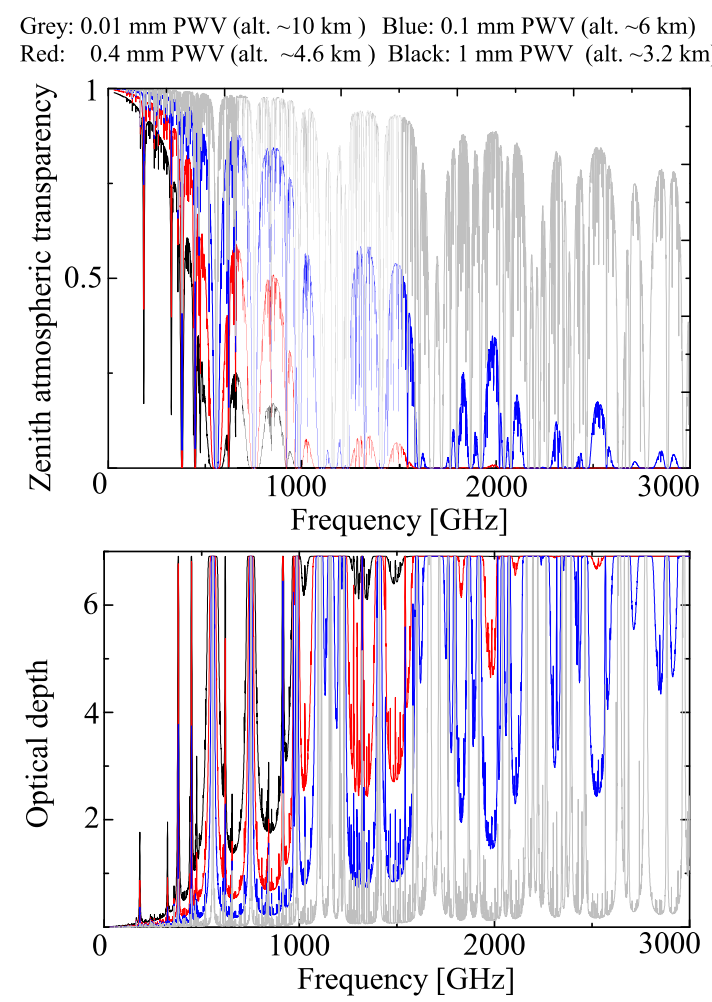

Fig.3 Atmospheric transmission as a function of frequency calculated with a radiative transfer model for the four different precipitable water vapor (PWV) conditions of $0.01,0.1,0.4$, and $1 \mathrm{~mm}$.

where $\tau$ is the optical depth of the sky, $T_{S K Y}$ is the temperature of the atmosphere, $T_{R X}$ is the receiver noise temperature, $T_{M I X}$ and $T_{I F}$ are the equivalent noise temperatures of the mixer detector and the IF chain system, respectively, $T_{R F}$ and $G_{R F}$ are the equivalent noise temperature and transparency brought by the beam-correcting devices in the receiver, optics, and antenna systems, and $G_{M I X}$ is the gain of the mixer. The equivalent noise temperature and band characteristics of the cooled amplifiers as well as those of the mixer detector play essential roles in achieving the high sensitivity and wide bandwidth of heterodyne receiver systems. The receiver noise temperature is generally evaluated according to the Callen-Welton formula with hot ( 300 K) and cold ( temperature of liquid nitrogen) blackbody radiation [10]. The optical depth $\tau$ even at a desert highland of $4 \mathrm{~km}$ in altitude is basically greater than unity above $1 \mathrm{THz}$ because of atmospheric loss from the absorption by water vapor as shown in Fig. 3, which had been the main bottleneck for ground-based THz-band observations in astronomy and earth and planetary atmospheric researches.

\section{Superconducting HEB Mixer}

\subsection{Basic Principles}

The first observation employing an InSb-semiconductorbased HEB mixer of an interstellar cloud was demonstrated in 1973 [11]. The thermal time constant of the InSb HEB mixer was $\sim 10^{-7} \mathrm{~s}$, corresponding to an IF bandwidth of 1 (a)

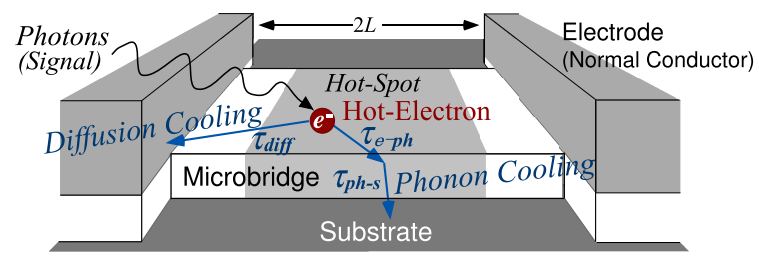

(b)

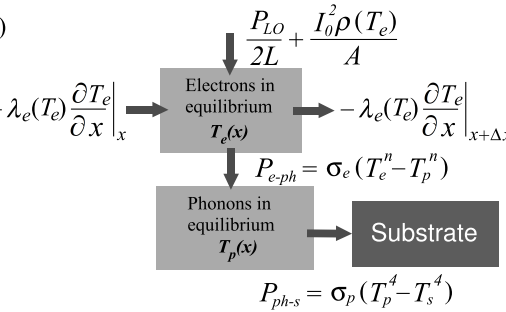

(c) $R$-T Characteristics

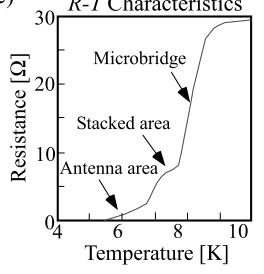

Fig. 4 Schematic drawings of the microbridge (a), the heat balance diagram (b), and the $R-T$ characteristics (c).

MHz. At present, superconducting HEB mixers employing thin and short-length microbridges made of low-temperature superconductors such as $\mathrm{NbN}$ and NbTiN hold the best prospect as heterodyne detectors in the $\mathrm{THz}$ frequency band. These kinds of superconducting mixers offer a high-speed bolometric response of several gigahertz through phonon cooling between the microbridge and the substrate and/or diffusion of hot electrons from the microbridge to the normal conductor electrodes [12], [13], as shown in Fig. 4.

Nearly a decade of improvements in the microbridge size, fabrication processes, and theoretical and design models have led to HEB mixers with sensitivities achieving quantum-noise level $\left(5-10 h v / k_{B}\right)$ at the several terahertz band. The upper limit of operational frequency for superconducting HEB mixers is not determined by the energy gap, unlike SIS mixers, because the HEB mixers work as transition-edge sensors by absorbing the photon at the superconductor microbridge.

The heat balance equations for a small segment along the microbridge ( $x$ axis), where the temperature distributions in the directions of the width and height of the microbridge are assumed to be uniform, can be expressed by

$$
\begin{aligned}
& \frac{P_{L O}}{2 L}+\frac{I_{0}^{2} \rho\left(T_{e}\right)}{A}+\frac{d}{d x}\left(\lambda_{e} \frac{d T_{e}}{d x}\right)=\sigma_{e}\left(T_{e}^{n}-T_{p}^{n}\right), \\
& \sigma_{e}\left(T_{e}^{n}-T_{p}^{n}\right)=\sigma_{p}\left(T_{p}^{4}-T_{s}^{4}\right),
\end{aligned}
$$

where $\lambda_{e}$ and $D$ are the thermal conductance and diffusion coefficient of electrons, respectively, $\sigma_{e}$ and $\sigma_{p}$ are the electron-phonon and phonon-substrate coupling efficiency, respectively, $2 L$ and $A$ are the length and cross-sectional area of the microbridge, respectively, $T_{e}, T_{p}$, and $T_{s}$ are the electron, phonon, and substrate temperatures, respectively, and $P_{L O}$ is the LO power absorbed in the microbridge. For $\mathrm{NbN} n$ is estimated to be $\sim 3.6$ experimentally. $\rho$ is the temperature-dependent local resistivity at the $x$-axis position along the microbridge.

The nonlinear resistance versus temperature $(R-T)$ characteristics have decisive influences on the sensitivity 
and gain bandwidth of the HEB mixers. The device's overall $R-T$ is determined by the superposition of several $R-T$ patterns of different $T_{c}$ 's of the superconducting microbridge and stack layers consisting of the superconducting film and normal conductors at the contact electrode and the antenna. The proximity between the thin superconductor film and the normal conductor and the heating effects caused by the DC and photon-induced currents reduce the transition temperature and broaden the transition width of the $R-T$ curve, resulting in degradation of nonlinearity of the $I-V$ curve of the HEB mixer.

By numerically calculating the balance Eqs. (3) and (4) based on the $R-T$ curve, we can derive the $T_{e}$ distributions along the microbridge for a bias current $I=I_{0}$, and then the $I-V$ curve and the conversion gain $G_{M I X}$ of a pumped HEB mixer at a bias point of $V=V_{0}$ can be calculated as follows [14], [15]:

$$
\begin{aligned}
G_{M I X}= & \frac{P_{I F}}{P_{S}}=\frac{2 I_{0}^{2} R_{L} C_{R F}^{2} P_{L O}}{\left(R_{0}+R_{L}\right)^{2}\left(1-C_{D C} I_{0}^{2} \frac{R_{L}-R_{0}}{R_{L}+R_{0}}\right)^{2}} \\
& \times \frac{1}{1+\left(2 \pi f_{I F} \times \tau_{M I X}\right)^{2}}\left(1-\left|\frac{Z_{\text {emb }}-Z_{\text {bridge }}}{Z_{\text {emb }}+Z_{\text {bridge }}}\right|^{2}\right),
\end{aligned}
$$

where $R_{0}=V_{0} / I_{0}$ denotes the device resistance caused by dc bias and the time-averaged RF irradiation, $R_{L}$ is the IF load resistance, and $P_{I F}, P_{S}$, and $P_{L O}$ are the output IF, absorbed $\mathrm{RF}$, and LO signal powers, respectively. The first term includes the electrothermal feedback effect where the current flow induced by a part of the IF signal reflected through the IF load further heats the microbridge. The final term is the coupling efficiency between the impedance of the microbridge, $Z_{\text {bridge }}\left(\sim R_{0}\right)$, and the embedding impedance of the mixer at the feed point, $Z_{e m b}$. $C_{D C}$ and $C_{R F}$ are the responsivities of $R_{0}$ with respect to the absorbed DC and RF power, respectively.

The small change in the resistance of the microbridge follows the relation $\Delta R=C_{D C} \Delta P_{D C}+C_{R F} \Delta P_{R F}$, so that $C_{D C}$ and $C_{R F}$ are derived by

$$
\begin{aligned}
& C_{R F}=\left.\frac{\partial R_{0}}{\partial P_{L O}}\right|_{\Delta P_{D C}=0}=\frac{R_{0}}{P_{D C}} \frac{\left(\frac{d V}{d I}\right)_{d c}-R_{0}}{\left(\frac{d V}{d I}\right)_{d c}+R_{0}}, \\
& C_{D C}=\left.\frac{\partial R_{0}}{\partial P_{D C}}\right|_{\Delta P_{L O}=0}=\frac{1}{\chi} C_{R F} .
\end{aligned}
$$

$\chi$ is the power exchange function with typical values of 1.0 3.0. $\tau_{M I X}$ is the relaxation time of the mixer, given by

$$
\begin{aligned}
\tau_{M I X} & =\tau_{e} \times\left(1-C_{D C} I_{0}^{2} \frac{R_{L}-R_{0}}{R_{L}+R_{0}}\right)^{-1}, \\
\tau_{e} & =\left(\frac{1}{\tau_{e-p h}+\left(C_{e} / C_{p}\right) \tau_{p h-s}}+\frac{1}{\tau_{\text {diff }}}\right)^{-1},
\end{aligned}
$$

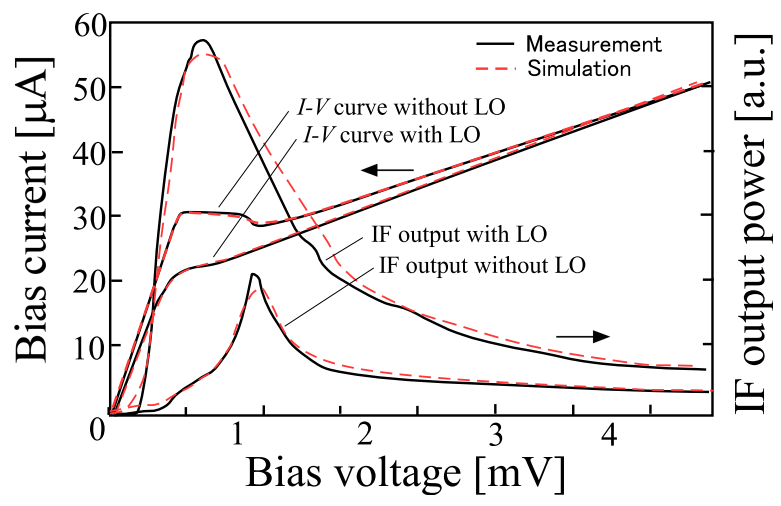

Fig. 5 Calculated and measured $I-V$ characteristics and IF output powers of an HEB mixer.

where $C_{e}$ and $C_{p}$ are the specific heats of the electrons and phonons, respectively. $\tau_{e}$ is the relaxation time of electrons, $\tau_{e-p h}$ is the time constant for electron-phonon interaction in the superconducting microbridge, and $\tau_{p h-s}$ is the time constant for the phonons to escape to the substrate. The diffusion time constant for hot electrons to escape to the electrodes on both sides of the microbridge, $\tau_{\text {diff }}$, is defined by $L^{2} / \pi^{2} D$ [16]. Examples of the calculated and measured $I-V$ curves and IF output powers are shown in Fig. 5.

For the DSB detection mode, the equivalent noise temperature of the HEB mixer, $T_{M I X, D S B}$, can be calculated by using [14]

$$
\begin{aligned}
T_{M I X, D S B} & =\left(T_{J n, D S B}^{\text {out }}+T_{T F n, D S B}^{\text {out }}\right) / 2 G_{M I X} \\
& =T_{J n, D S B}^{\text {in }}+T_{T F n, D S B}^{\text {in }} .
\end{aligned}
$$

$T_{J n, D S B}^{\text {out }}$ is the Johnson noise, and $T_{J n, D S B}^{i n}$ is expressed by

$$
T_{J n, D S B}^{i n}\left(I_{0}, P_{L O}\right)=\int_{-L}^{L} \frac{\rho\left(T_{e}, I_{0}, x\right) T_{e}}{I_{0}^{2} C_{R F}^{2} P_{L O} A} d x .
$$

$T_{T F n, D S B}^{\text {out }}$ is the temperature fluctuation noise, and $T_{T F n, D S B}^{i n}$ is expressed by

$$
\begin{aligned}
T_{T F n, D S B}^{i n}\left(I_{0}, P_{L O}\right)= & \int_{-L}^{L} \frac{1}{C_{R F}^{2} P_{L O}}\left(\frac{\partial \rho\left(T_{e}, I_{0}, x\right)}{\partial T_{e}}\right)^{2} \\
& \times \frac{T_{e}^{2} \tau_{e}}{C_{e} A^{3}} d x .
\end{aligned}
$$

Note that the electron temperature $T_{e}$ derived by solving the heat balance equations [Eqs. (3) and (4)] is a function of $I_{0}$ and $x$.

The HEB mixer is operated under the resistive transition state between superconducting and normal conducting states of the microbridge by optimizing the bias voltage and the LO power precisely. One of the keys to improving the sensitivity and bandwidth of an HEB mixer is the optimization of $\tau_{\text {diff }}$ and $\tau_{p h-s} . \tau_{\text {diff }}$ can be improved by selecting the material of the microbridge, shortening its length $L$, increasing its thickness, and depositing high-quality contact electrode pads onto the microbridge through in situ fabrication processes and/or $\tau_{p h-s}$ can be improved by acoustically 
matching the interface between the superconducting microbridge and the substrate with a buffer layer and by reducing the thickness of the microbridge.

The minimal length of the microbridge is restricted by the technical limitations of the electron-beam (EB) lithography and etching technologies. The thickness of the microbridge cannot be extremely reduced to avoid decreasing the critical temperature $\left(T_{c}\right)$ and broadening the width of the $R-T$ transition as this would degrade the nonlinear $I-V$ characteristics of the superconducting HEB mixer. However, if the microbridge is too thick, matching between $Z_{e m b}$ and the low $Z_{\text {bridge }}$ becomes difficult, degrading the phonon cooling effects. Owing to the small volume of the superconducting microbridge, the $\mathrm{LO}$ power required for $\mathrm{RF} / \mathrm{LO}$ mixing and suppression of the superconducting hysteresis current of a typical HEB mixer is about several tens of nanowatts, which is orders of magnitude lower than those required for SIS and Schottkey barrier diode (SBD) mixers. This is one of the crucial advantage of HEB mixers. The dimensions and material of the HEB mixer are empirically determined by considering these design trade-off between diffusion and phonon cooling effects. The most widely used superconducting mircobrides are of $\mathrm{NbN}$ and $\mathrm{NbTiN}$ at present. The typical dimensions of the microbridge are 100-300 nm long, 30-100 ̊ thick, and $\sim 1 \mu \mathrm{m}$ wide.

The gain bandwidth of most phonon-cooled NbN HEB mixers is limited by the escape time of nonequilibrium phonons from the superconducting microbridge to the substrate. The typical gain bandwidth reaches $\sim 5 \mathrm{GHz}$ by using a 3.5-nm-thick $\mathrm{NbN}$ microbridge grown on a crystalline substrate such as $\mathrm{MgO}$ and highly resistive $\mathrm{Si}$ preheated to $\sim 800^{\circ} \mathrm{C}$. Recently, the Moscow State Pedagogical University (MPSU) group improved both phonon and diffusion cooling devices by shortening the length of the $\mathrm{NbN}$ microbridge to $0.12 \mu \mathrm{m}$ and introducing an in situ process for better connection between the microbridge and the contact electrodes [17], [18]. They achieved a 7-GHz noise bandwidth and a simultaneously equivalent noise temperature (DSB) of $600 \mathrm{~K}$ at $2.5 \mathrm{THz}$ experimentally, where $\tau_{\text {diff }}$ and $\tau_{p h-s}$ are estimated to be comparable to $40 \mathrm{ps}$. The University of Tokyo group developed waveguide-type low-noise 0.8 - and 1.5-THz-band HEB mixers by employing a relatively thick NbTiN microbridge fabricated in an in situ process, for which the corrected receiver noise temperatures are 220 and $250 \mathrm{~K}$ with the IF $3 \mathrm{~dB}$-rolloff bandwidths of 1.5 and $2.5 \mathrm{GHz}$, respectively [19]. The performances of recent HEB mixers are shown in Fig. 6 with comparisons to the sensitivity of SIS mixers and semiconductor SBD mixers.

\subsection{Fabrication Process}

Currently, $\mathrm{NbN}$ and NbTiN films are the most commonly used for the superconducting microbridges mentioned above. The Harvard-Smithonian Center for Astrophysics and Jet Propulsion Laboratory (JPL), California Institute of Technology, group succeeded in fabricating a NbTiN microbridge on a crystalline quartz substrate pre-

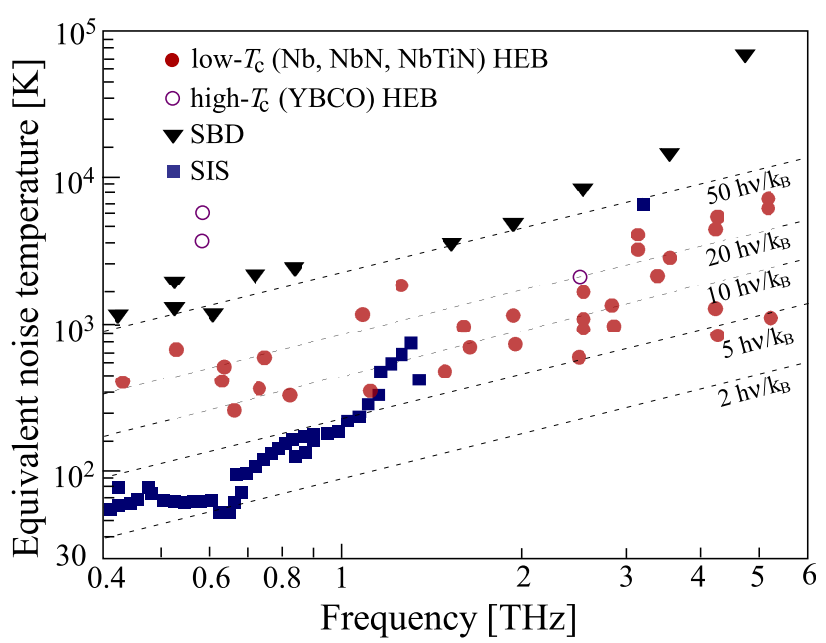

Fig. 6 Equivalent noise temperatures in DSB mode of the superconducting HEB, SIS, and semiconductor SBD mixers (e.g. [19]-[21]).

heated to $375^{\circ} \mathrm{C}$ over a 20 -nm-thick AlN buffer layer and demonstrated for the first time the scientific significance of the low-noise NbTiN HEB mixer for observation of the CO $J=7 \rightarrow 6$ line from an astronomical source, IRC +10216 , with the Submillimeter Telescope in Arizona [22]. Here we present one of our in situ fabrication processes for HEB mixers employing a NbTiN microbridge at the University of Tokyo [19], [23]-[25].

We developed a multideposition system exclusively for in situ fabrication of HEB mixers. In this system, to control the thickness of the microbridge precisely, a slow deposition rate of $1 \AA / \mathrm{s}$ is achieved under high vacuum condition by using a helicon plasma sputtering technique. The sputtering chamber is connected to the electron-beam evaporation chamber via a load lock chamber, which allows us to deposit a 2-nm-thick Ti interface layer and a 100-nm-thick $\mathrm{Au}$ electrode layer on the sputtered NbTiN layer without breaking the vacuum. This in situ technique is essential for obtaining a good contact at the $\mathrm{NbTiN} / \mathrm{Ti} / \mathrm{Au}$ interface as it prevents natural oxidation. The 4- to 12-nm-thick NbTiN films are deposited on a Z-cut crystalline $\mathrm{SiO}_{2}$ substrate for a waveguide-type mixer chip or on a nondoped highresistivity floating zone $\mathrm{Si}$ substrate for a twin-slot-antennatype mixer chip, in which the NbTi target is sputtered with an mixture of $\mathrm{N}_{2}$ and $\mathrm{Ar}$ inlet gases at room temperature. Before this process an AlN buffer layer is sputtered on the substrate in the multideposition system as necessary [26].

The stress, resistivity, and $T_{c}$ of NbTiN are crucial parameters for determining the properties of the HEB mixer. The stress and resistivity depend strongly on the crystalline structures of the sputtered film, which are very sensitive to the total process pressure. To keep the pressure constant, the inlet mass flow velocity of $\mathrm{N}_{2}$ and Ar gas, the exhaust velocity from a molecular turbo evacuation system, and the discharge current are precisely tuned according to a process simulation [27]. In this simulation model fine changes of the sputtering condition caused by the erosion of the NbTi target are also considered. The dimensions of the microbridge 


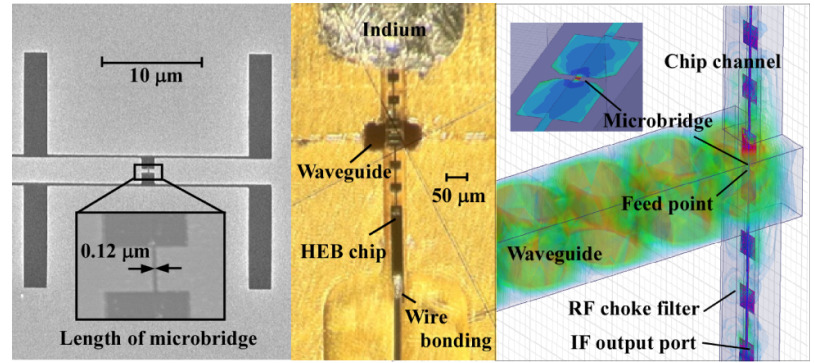

Fig. 7 SEM photograph of the quasi-optical twin-slot antenna and the microbridge part of an HEB mixer (left), an optical micrograph of an HEB mixer chip mounted in a chip channel (center), and an HFSS simulation at the waveguide-to-microstrip transition structure of the HEB mixer (right).

are defined by using EB lithography and subsequently by dry-etching of the Au layer on the NbTiN/Ti layer with an inductively coupling plasma (ICP) system. The Ti layer works as an etching stopper. In this procedure, the spatial resolution of the EB lithography and the accuracy of the etching rate and the redeposition of the etched byproducts on the sidewall of the Au electrodes in the ICP etching processes determine the effective length of the microbridge of the HEB mixer. For twin-slot-antenna-type mixers, coplanar-type choke filters and slot antenna patterns are defined by photolithography as shown in Fig. 7 and then a low-loss normal conductor at the $\mathrm{THz}$ band (e.g., Au or $\mathrm{Al}$ ) is deposited. After the subsequent lift-off process, for waveguide-type mixers, the back side of the substrate is polished, and then the substrate is chipped with a dicing machine.

Finally, the chips are mounted on a mixer block and the IF port on the chip is linked via bonding wires to an IF microstrip line on a Duroid substrate integrated into a mixer block as shown in Fig. 7. The quasi-optical mixer chip is attached to the bottom surface of a hyper- or extendedhemisphere lens made of nondoped high-resistivity Si with an antireflection (AR) coating. The waveguide probe that couples the input signal can be designed with the aid of 3D EM field simulator such as HFSS(TM) as shown in Fig. 7. The probe feed is optimized so that it matches the microbridge impedance. For example, twin-slot antenna type mixers can be redesigned with a boundary element method and method of moments numerical technologies on the basis of the conventional model [28], [29]. The frequency responses and co- to cross-polarization of the HEB mixers are checked by using an FTS system.

\subsection{Mixer Mount Design}

The beam pattern of a mixer detector, including its beam size, the side-lobe level, and the polarization, is one of the essential factors for astronomical observations. In the millimeter to submillimeter wave band, corrugated horn antennas are widely utilized because of their well-defined beam pattern. Above $1 \mathrm{THz}$, the usual diagonal horns are often adopted because of their ease of fabrication. Above the 2-THz band, for which micromachining of the waveguide structures becomes very difficult, quasi-optical log-spiral and double-slot planar antennas on hyper- and extended-hemispherical lenses are comparatively well used. In waveguide-type HEB mixers, the electromagnetic field of the fundamental $\mathrm{TE}_{10}$ waveguide mode is coupled to the microbridge at the feed point of the waveguide-to-microstrip transition area, and the dimension of the substrate of the HEB mixer chip and the chip channel slot are designed so as to suppress RF leakage through surface wave modes in the substrate. For the design of optical components, various commercial simulators such as GRASP are widely utilized [30].

The University of Cologne has developed a highsensitive 2.5-THz-band NbTiN HEB mixer integrated onto a conventional waveguide block machined for the German REceiver for Astronomy at Terahertz frequencies (GREAT) on the Stratospheric Observatory For Infrared Astronomy (SOFIA) [31]. To obtain the good lattice matching at the interface between the microbridge and substrate, the $\mathrm{NbTiN}$ microbridge is fabricated onto a $2-\mu \mathrm{m}$-thick low-stress silicon nitrite ( $\mathrm{SiN}$ ) membrane deposited on a 500- $\mu$ m-thick silicon wafer for easier processing. Finally, this silicon wafer is back-etched. The DSB equivalent receiver noise temperatures for 1.9- and 2.53-THz bands achieved $\sim 1500 \mathrm{~K}$.

To produce waveguide circuits with fine accuracy and high reproducibility, JPL of NASA tackled the novel development of a 2.7-THz waveguide-based NbN HEB mixer [32]. They deposited a thin $\mathrm{MgO}$ buffer layer onto silicon on an insulator wafer (SOI) consisting of a $2.5-\mu \mathrm{m}$ highresistive Si layer/2- $\mu \mathrm{m}$ oxide layer/400- $\mu \mathrm{m}$ handling wafer. $\mathrm{The} \mathrm{NbN}$ film is sputtered onto the $\mathrm{MgO}$ layer. The channel slot structure for the mixer chip with $22 \mu \mathrm{m}$ width and $3 \mu \mathrm{m}$ thickness is perpendicular to a half-height waveguide transition (of dimensions of $78 \times 19 \mu \mathrm{m}$ ) to a metal Pickett Potter horn manufactured commercially by electroforming. The slot channel is composed of the stack of three gold layers of 5,4 , and $5 \mu \mathrm{m}$ thickness, which are fabricated by means of deep UV photolithography with the thick GKR4400 resist and a microplating technique. The noise temperature of this waveguide $\mathrm{NbN}$ mixer achieved $965 \mathrm{~K}$ at $2.74 \mathrm{THz}$.

The group of advanced receiver development of Chalmers University of Technology developed a balance waveguide HEB mixer for the $1.25-1.39 \mathrm{THz}$ band [33] The mixer mount block consists of two NbN HEB mixer chips and a waveguide $3-\mathrm{dB} 90^{\circ} \mathrm{RF}$ hybrid fabricated by using an advanced photolithographic microfabrication technology of thick SU-8 photoresist combined with copper electroplating. This process gives fabrication reproducibility with an accuracy of better than $2 \mu \mathrm{m}$ and a surface accuracy of the waveguide wall of $<100 \mathrm{~nm}$ rms. The mixer is cooled to $4 \mathrm{~K}$ with an assembly bracket in the closedcycle cryocooler of the Swedish heterodyne Facility Instrument (SHeFI). The receiver noise temperature and spectroscopic Allan time achieved were $<1200 \mathrm{~K}$ and $>200 \mathrm{~s}$, respectively. In 2008, they succeeded in observing the CO $J=11 \rightarrow 10$ line toward the AGB star CW Leo with this SHeFI receiver installed in the Atacama Pathfinder Exper- 
iment (APEX) telescope located at Liano de Chajnantor in Northern Chile (at an altitude of $5105 \mathrm{~m}$ ). They also developed a balance waveguide HEB mixer for the 1.6-2.0 $\mathrm{THz}$ band [34]. The mixer block is composed of corrugated horns for RF and LO signals, a waveguide 3-dB 90 RF hybrid with a minimum slot dimension of $21 \mu \mathrm{m}$, two HEB mixers employing $\mathrm{NbN}$ microbridges grown on a preheated $\left(700^{\circ} \mathrm{C}\right) 14 \times 14 \mathrm{~mm}^{2}$ double-bonded SOI substrate, and a 3-dB $90^{\circ}$ IF hybrid circuit. The novel mixer chip supported by the SOI frame facilitates handling of the chip and electrical access. Such a technical approach may break ground for multipixel waveguide receivers in the several $\mathrm{THz}$ band.

\subsection{Operations with $4 \mathrm{~K}$ Refrigerators}

$\mathrm{NbN}$ and NbTiN HEB mixers are usually cooled with a commercially available $4 \mathrm{~K}$ cryocooler (e.g., an infrared dewar cooled with liquid helium or a mechanical closed-cycle $4 \mathrm{~K}$ cryocooler). Mechanical closed-cycle Gifford MacMahorn (GM) and pulse-tube (PT) $4 \mathrm{~K}$ cryocoolers are very useful for long-term field observations with superconducting detectors. However, temperature fluctuations of the PT cryostat and mechanical vibrations of the GM cryostat induce periodic changes in the gain of the HEB mixer, which degrades the Allan variance time of the receiver system. For example, the PT $4 \mathrm{~K}$ cryocooler (SPR-062B) from Sumitomo Heavy Industries, Ltd., has a cooling capacity of $0.5 \mathrm{~W}$ and an amplitude of mechanical vibration of $<3 \mu \mathrm{m}$. The peak-to-peak temperature fluctuation of the PT cryostat induced by the inner He gas circulation is $320 \mathrm{mK}$, which is greater than that of the GM $4 \mathrm{~K}$ cryocooler. This temperature fluctuation is not negligible compared to the width of the transition temperature between the superconducting state and normal conducting state of the microbridge of the HEB mixer. Inserting buffer materials with very high heat capacity at low temperature between the HEB mixer and cold head of the cryocooler can reduce the temperature vibrations [25]. Another interesting method is to stabilize the bias current of the HEB mixer directly by injecting microwave radiation from the IF port [35].

\section{Observations with HEB Mixers}

In 1998, the Harvard-Smithsonian Center for Astrophysics group succeeded in observing a spectral line of the $J=$ $7 \rightarrow 6$ transition of $\mathrm{CO}$ at $806.651 \mathrm{GHz}$ toward the Orion Kleinmann-Low (Orion-KL) nebula with massive star-forming regions using a waveguide-type $\mathrm{NbN} \mathrm{HEB}$ mixer detector with the 10-m Heinrich Herz Telescope (HHT) on Mt. Graham, Arizona [36]. In addition, the same group observed a $J=11 \rightarrow 10$ transition line at $1.267 \mathrm{THz}$ toward M17 and a $J=13 \rightarrow 12$ transition line at $1.497 \mathrm{THz}$ of ${ }^{12} \mathrm{CO}$ toward Orion-KL nebula with the $0.8-\mathrm{m}$ receiver lab telescope located at an elevation of $5525 \mathrm{~m}$ in Chile in 2004 [37] and successfully obtained a two-dimensional image of ${ }^{12} \mathrm{CO} J=9 \rightarrow 8(1.037 \mathrm{THz})$ toward the Orion-KL nebula with a spatial resolution of $84^{\prime \prime}$ in 2006 [38]. In these observations the mixers were cooled to $4.2 \mathrm{~K}$ with a liquidhelium-cooled dewar.

The Herschel-Heterodyne Instrument for the FarInfrared (HIFI) is the receiver system onboard the Herschel Space Observatory launched in 2009; it employs 1.4-1.7 and 1.7-1.9 THz band NbN HEB mixers [39], [40]. The HIFI spectral surveys presented the widest spectral coverage ever observed in these frequency ranges, covering the transition lines of $\mathrm{SO}, \mathrm{H}_{2} \mathrm{~S}, \mathrm{HCN}, \mathrm{H}_{2} \mathrm{O}, \mathrm{CH}_{3} \mathrm{OH}$, and so on toward the Orion-KL nebula [41]. The HIFI GOT C+ Galactic plane [CII] spectral survey revealed the bright emission of [CII] at $1900.5469 \mathrm{GHz}$ in the Scutum-Crux spiral arm tangencies as shock compression of the warm ionized medium induced by spiral density waves [42].

SOFIA is an airborne observatory that comprises a modified Boeing 747SP with a 2.7-m telescope. SOFIA employs a photometer, near-, mid-, and far-infrared cameras, infrared spectrometers, and the GREAT heterodyne spectrometer, covering wavelengths from $0.3 \mu \mathrm{m}$ to $1.6 \mathrm{~mm}$ [43], [44]. GREAT uses waveguide HEB mixer detectors cooled at $4 \mathrm{~K}$ with cryogenic amplifiers in a liquid-heliumcooled cryostat, giving a hold time of $>15 \mathrm{~h}$ [45]. The LO source system consists of solid-state cascading multiplier chains from Virginia Diodes, Inc. Utilized as backends are an acousto-optical spectrometer covering two 4-GHz-wide bands, a chirp transform spectrometer with 220-MHz bandwidth and 56-kHz resolution [46], and a XFFTS.

In 2011, observations of many interstellar gaseous species such as carbon monoxide $(\mathrm{CO})$, mercapto radicals $(\mathrm{SH})$, hydroxyl radicals $(\mathrm{OH})$, deuterated hydroxyl radicals (OD), ammonia $\left(\mathrm{NH}_{3}\right)$, ionized carbon [C II], and so on were made toward various astronomical targets with the SOFIA GREAT instrument. The $\mathrm{OH}$ radical plays a crucial role in the chemical balance in the interstellar medium (ISM) and planetary atmospheres. For understanding the chemical reaction network of the ISM, observations at $2.514 \mathrm{THz}$ for ${ }^{2} \Pi_{3 / 2} J=5 / 2 \leftarrow 3 / 2$ and $1.837 \mathrm{THz}$ for ${ }^{2} \Pi_{3 / 2} J=$ $3 / 2 \leftarrow 1 / 2$ transition lines of $\mathrm{OH}$ hyperfine structure and $2.494 \mathrm{THz}$ for ${ }^{2} \Pi_{3 / 2} J=5 / 2 \leftarrow 3 / 2$ transitions lines of ${ }^{18} \mathrm{OH}$ were performed toward the diffuse clouds W49N, W51, and G34.26+015 in the Carina-Sagittarius spiral arm [47]. The $3_{2+} \rightarrow 2_{2-}$ transition lines at 1810.379971 $\mathrm{GHz}$ of $\mathrm{NH}_{3}$, which is comparatively free from freezing out onto dust grains in the initial cold phase of molecular cloud cores, was observed toward the high-mass-starforming clumps as a tracer of dynamical infall motion in the early stage of star formation [48]. To understand the formation mechanism of water in the interstellar medium, the ${ }^{2} \Pi_{3 / 2} J=5 / 2 \rightarrow 3 / 2, l=-1 \rightarrow+1$ multiplet line of OD was observed at the $1.3915-\mathrm{THz}$ band toward a lowmass protostar. The inferred high OD/HDO ratio, which is higher than predicted by standard chemical models including gas-phase and dust chemistries, implies that exothermic $\mathrm{OH}+\mathrm{D}$ exchange promotes the fractionation of $\mathrm{OH}$ relative to water in the gas phase via dissociative recombination reprocessing of $\mathrm{H}_{2} \mathrm{DO}^{+}$[49]. The existing form of sulfur is considered to be extremely varied through complex chemi- 
cal reactions in the gas phase and on the dust grain surface in the ISM. Thus an accurate abundance value for sulfur in the ISM is a missing piece of the puzzle. To understand the abundance of sulfur-bearing hydrides in the ISM, the ${ }^{2} \Pi_{3 / 2} J=5 / 2 \leftarrow 3 / 2$ lambda doublet of SH was observed at the $1.383-\mathrm{THz}$ band along the line of sight toward the submillimeter continuum source W49N with the GREAT instrument. The derived $\mathrm{SH} / \mathrm{H}_{2} \mathrm{~S}$ abundance ratio was lower than the value predicted by standard models including the effects of the photodissociation region, turbulent dissipation region, or continuous- or jump-type shocks, which may suggest enhancement of the endothermic neutral-neutral reaction $\mathrm{SH}+\mathrm{H}_{2} \rightarrow \mathrm{H}_{2} \mathrm{~S}+\mathrm{H}$ in the ISM [50]. [CII] lines were observed also toward planetary nebula such as the Ring Nebula in Lyra (NGC6720), suggesting that the [CII] is a better tracer of circumstellar material and other evolved planetary nebulae than $\mathrm{CO}$ because the bulk of the element carbon is divided roughly equally between [CII] and [CI] [51].

The CO N+ Deuterium Observations Receiver (CONDOR) with a waveguide NbTiN HEB mixer was installed on the 10-m APEX telescope. CONDOR employs a closedcycle PT cryocooler and solid-state LO sources manufactured by Radiometer Physics $\mathrm{GmbH}$ and Virginia Diode, Inc. With CONDOR, observations of high- $J$ CO lines, the transition line of [NII], and the ground transition of para$\mathrm{H}_{2} \mathrm{D}^{+}$, which are good tracers of hot dense gas, ionized diffuse gas, and cold dense gas, respectively, were demonstrated toward Orion FIR4 in 2006 [52]. The University of Tokyo group started astronomical observation by installing their own 0.9- and 1.3-THz-band waveguide-type NbTiN HEB mixers onto the 10-m Atacama Submillimeter Telescope Experiment (ASTE) located at Pampa La Bola in northern Chile (at an altitude of $4860 \mathrm{~m}$ ) in 2011 [19].

The Terahertz and submillimeter Limb Sounder (TELIS), being developed by the Space Research Organization of the Netherlands, the Rutherford Appleton Aerospace Center in the United Kingdom, and the German Aerospace Center, is preparing 0.5-, 0.6-, and 1.8-THz-band heterodyne receivers including NbN HEB mixer coolers with an IRlabs HDL-5 dewar [53]. Observation during the flight duration of about $24 \mathrm{~h}$ will provide important information on the diurnal variation of key atmospheric short-lived radicals such as $\mathrm{OH}, \mathrm{HO}_{2}, \mathrm{ClO}$, and $\mathrm{BrO}$ together with stable constituents such as $\mathrm{O}_{3}, \mathrm{HCl}$, and $\mathrm{HOCl}$.

\section{Outlook}

In this decade and beyond, further development of highly sensitive superconducting HEB mixers will drive THz-band astronomy and its widespread application. Future challenges for HEB mixers include improving the sensitivity and bandwidth by optimizing the size of the microbridge, along with refining the fabrication yield, simplifying the handling of the mixer chips and mounts and of the alignment of the optical components, and stabilizing the HEB mixer receivers.

We are currently developing a waveguide-type HEB mixer employing a diagonal horn for the $1.8-2.0 \mathrm{THz}$ band, where the dimensions of a NbTiN microbridge fabricated by our in situ technique are optimized on the basis of the model mentioned in Section 3.1. The observational targets for this frequency band are $\mathrm{OH}$ radicals, which are the central prooxidant in the chemical reaction network in the earth's atmosphere and planetary atmospheres; lines from [OI] and [C II], which are the basic coolants of the interstellar medium; and other complex and high- $J$ molecules. The designed chip width and thickness of the mixer are $44 \mu \mathrm{m}$ and $19 \mu \mathrm{m}$, respectively. The establishment of such microfabrication processes will also support the mass production of the THzband heterodyne receivers studied in the ALMA project collectively referred to as Band 11. The observational targets of Band 11, which covers 1.0-1.6 THz, are [C II] lines from intermediate redshift galaxies $(0.3<\mathrm{Z}<1)$; [NII] lines; deuterated nitrogen-bearing molecules such as $\mathrm{N}_{2} \mathrm{D}^{+}$and $\mathrm{NH}_{2} \mathrm{D}$, which are good tracers of dense core regions; high- $J$ lines emitted from UV and shock-heated gases in star-forming regions; para- $\mathrm{H}_{2} \mathrm{D}(1370 \mathrm{GHz})$ and ortho- $\mathrm{D}_{2} \mathrm{H}^{+}(1476 \mathrm{GHz})$; and so on. The ortho/para ratio of $\mathrm{H}_{2} \mathrm{D}^{+}$provides us with crucial information about the physical conditions of dense clouds where deuterium fractionation and the ortho/para ratio of $\mathrm{H}_{2}$ molecules proceed through the exothermic protondeuteron exchange reaction $\mathrm{H}^{+}+\mathrm{HD} \rightarrow \mathrm{H}_{2} \mathrm{D}^{+}+\mathrm{H}_{2}$.

In DSB heterodyne detection, spectral lines in the image band often interfere with spectral lines in the signal band of interest. In addition, the noise performance of DSB receivers is often degraded by the atmospheric equivalent noise from the image band. To overcome these problems, sideband separation (2SB) and balanced waveguide mixers are also worth considering in the $\mathrm{THz}$ band by following up on the surface roughness of the waveguide wall and fabrication processing of mount block components. These unrelenting approaches will enable developments of multipixel receiver systems, as are being planned for upGREAT instruments including $2 \times 7$ pixels for $1.9-2.5 \mathrm{THz}$ and $1 \times 7$ pixels for $4.7 \mathrm{THz}$ [45] covering the fine structure line of atomic oxygen [OI], which will allow for highly efficient two-dimensional imaging observations.

For THz-band imaging based on heterodyne spectroscopy in astronomy, the quantum cascade laser (QCL) is one of the most promising compact, high-power $\mathrm{CW}$ LO sources (e.g. [54], [55]). Recently, 1-5 THz band submilliwatt-class $\mathrm{CW}$ emissions have been demonstrated using metal-metal (MM) and semi-insulation-surfaceplasmon (SI-SP) waveguide type QCLs based on chirped superlattice (CSL), bound-to-continuum (BTC), and resonant phonon (RP) active regions [55]-[58]. A phase-locking system with an HEB mixer can be a useful tool for precisely stabilizing the frequency of emission of the QCLs [59][62]. Recently, spectroscopic observations of methanol $\left(\mathrm{CH}_{3} \mathrm{OH}\right)$ have been successfully demonstrated with $\mathrm{NbN}$ HEB mixer receivers at $2.918,3.5$, and $4.7 \mathrm{THz}$ by using a single-mode third-order distributed feedback (DFB) grating and MM waveguide type RP-QCLs, in which pressurebroadened Lorenzian line profiles were reproducibly re- 
solved with an FFTS with a bandwidth of $1.5 \mathrm{GHz}$ and 8192 frequency channels developed by MPIfR in Bonn [63]-[65]. The DFB QCL has a low divergent field beam that couples efficiently with the beam of the HEB mixer detector [66]. These combined heterodyne receiver systems with superconducting HEB mixers and QCLs will open up laboratory spectroscopies of molecular, atomic, and plasma gaseous species as well as THz-band astronomy with multipixel heterodyne receivers and remote sensing of earth's atmosphere and other planetary atmospheres.

The strengthening of the strategic aircraft- and satellitebased observatories also plays a key part in further development of highly efficient THz-band spectroscopic sensing. Such flight missions require tough sensors that are resistant to the harsh operating conditions, where the sensors are exposed to repeated cooling/heating cycles in prior reliability verification tests. The heavy-duty superconducting HEB mixer employing a thick NbTiN microbridge is one of the most promising THz-band heterodyne sensors for use in such field missions.

\section{References}

[1] C. L. Bennett, D. J. Fixsen, G. Hinshaw, J. C. Mather, S. H. Moseley, E. L. Wright, R. E. Eplee, Jr., J. Gales, T. Hewagama, R. B. Isaacman, R. A. Shafer, and K. Turpie, "Morphology of the interstellar cooling lines detected by COBE," Astrophys. J., vol.434, no.2, pp.587-598, Oct. 1994.

[2] D. J. Hollenbach and A. G. G. M. Tielens, "Dense photodissociation regions (PDRs)," Annu. Rev. Astron. Astrophys., vol.35, no.1, pp.179-215, Sept. 1997.

[3] D. C. Mattis and J. Bardeen, "Theory of the anomalous skin effect in normal and superconducting metals," Phys. Rev., vol.111, no.2, pp.412-417, July 1958.

[4] Y. Uzawa, M. Kroug, T. Kojima, M. Takeda, M. Candotti, Y. Fujii, K. Kaneko, W. Shan, T. Noguchi, and Z. Wang, "A sensitive ALMA Band 10 SIS receiver engineering model," Supercond. Sci. Technol., vol.22, no.11, pp.114002, Nov. 2009.

[5] J. J. A. Baselmans, A. Baryshev, S. F. Reker, M. Hajenius, J. R. Gao, T. M. Klapwijk, B. Voronov, and G. Gol'tsman, "Influence of the direct response on the heterodyne sensitivity of hot electron bolometer mixers," J. Appl. Phys., vol.100, no.8, pp.084510, Oct. 2006.

[6] J. J. A. Baselmans, A. Baryshev, S. F. Reker, M. Hajenius, J. R. Gao, T. M. Klapwijk, Y. Vachtomin, S. Maslennikov, S. Antipov, B. Voronov, and G. Gol'tsman, "Direct detection effect in small volume hot electron bolometer mixers," Appl. Phys. Lett., vol.86, no.16, pp.163503, Apr. 2005.

[7] M. Hajenius, J. J. A. Baselmans, A. Baryshev, J. R. Gao, T. M. Klapwijk, J. W. Kooi, W. Jellema, and Z. Q. Yang, "Full characterization and analysis of a terahertz heterodyne receiver based on a $\mathrm{NbN}$ hot electron bolometer," J. Appl. Phys., vol.100, no.7, pp.074507, Oct. 2006.

[8] T. W. Crowe, J. L. Hesler, S. A. Retzloff, C. Pouzou, and G. S. Schoenthal, "Solid-state LO sources for greater than 2THz," 22nd Int. Symp. Space Terahertz Technol., pp.209-212, 2011.

[9] B. Klein, S. Hochgürtel, I. Krämer, A. Bell, K. Meyer, and R. Güsten, "High-resolution wide-band fast Fourier transform spectrometers," Astron. Astrophys., vol.542, pp.L3, June 2012.

[10] A. K. Kerr, "Suggestions for revised definitions of noise quantities, including quantum effects," IEEE Trans. Microw. Theory Tech., vol.47, no.3, pp.325-329, Mar. 1999.

[11] T. G. Phillips and K. B. Jefferts, "A low temperature bolometer heterodyne receiver for millimeter wave astronomy," Rev. Sci. Instrum., vol.44, no.8, pp.1009-1014, Aug. 1973.

[12] E. M. Gershenzon, G. N. Gol'tsman. I. G. Gogidze. Y. P. Gusev. A. I. Elant'ev. B. S. Karasik, and A. D. Semenov, "Millimeter and submillimeter range mixer based on electronic heating of superconducting films in the resistive state," Sov. Phys. Superconductivity, vol.3, pp.1582-1597, Oct. 1990.

[13] D. E. Prober, "Superconducting terahertz mixer using a transitionedge microbolometer," Appl. Phys. Lett., vol.62, no.17, pp.21192121, Apr. 1993.

[14] P. Khosropanah, H. Merkel, S. Yngvesson, A. Adam, S. Cherednichenko, and E. Kollberg, "A distributed device model for phononcooled HEB mixers predicting IV characteristics, gain, noise and IF bandwidth," 11th Int. Symp. Space Terahertz Technol., pp.465-479, 2000

[15] H. Merkel, P. Khosropanah, D. Wilms-Floet, P. Yagoubov, and E. Kollberg, "Conversion gain and fluctuation noise of phonon-cooled HEB in hot spot regime," IEEE Trans. Terahertz Sci. Technol., vol.48, no.4, pp.690-699, Apr. 2000.

[16] P. J. Burke, R. J. Schoelkopf, D. E. Prober, A. Skalare, B. S. Karasik, M. C. Gaidis, W. R. McGrath, B. Bumble, and H. G. LeDuc, "Mixing and noise in diffusion and phonon cooled superconducting hotelectron bolometers," J. Appl. Phys., vol.85, no.3, pp.1644-1653, Feb. 1999.

[17] S. Ryabchun, M. Finkel, I. Tretyakov, A. Maslennikova, N. Kaurova, B. Voronov, and G. Gol'tsman, "Next generation of hot-electron bolometer mixers for future heterodyne missions," 22st Int. Symp. Space Terahertz Technol., pp.187, 2011.

[18] I. V. Tretyakov, M. I. Finkel, S. A. Ryabchun, A. I. Kardakova, S. V. Seliverstov, D. V. Petrenko, and G. N. Goltsman, "Hot-electron bolometer mixers with in situ contacts," Radiophys Quantum El., vol.56, no.8-9, pp.591-598, Jan. 2014.

[19] T. Shiino, R. Furuya, T. Soma, T. Sakai, Y. Watanabe, N. Sakai, L. Jiang, O. Ohguchi, H. Maezawa, T. Yamakura, Y. Irimajiri, and S. Yamamoto, "The 0.9 and $1.3 \mathrm{THz}$ superconducting HEB mixer receiver for the ASTE $10 \mathrm{~m}$ telescope," 23rd Int. Symp. Space Terahertz Technol., pp.76, 2012.

[20] H.-W. Huber, IEEE J. Sel. Top. Quantum Electron., vol.14, no.2, pp.378-391, Mar--Apr. 2008.

[21] R. G. Ladret, A. F. Degardin, and A. J. Kreisler, "Nanopatterning and hot spot modeling of YBCO ultrathin film constrictions for $\mathrm{THz}$ mixers," IEEE Trans. Appl. Supercond., vol.23, no.3, pp.2300305, June 2013.

[22] C. E. Tong, J. Stern, K. Megerian, H. LeDuc, T. K. Sridharan, H. Gibson, and R. Blundell, "A low-noise NbTiN hot electron bolometer mixer," 12th Int. Symp. Space Terahertz Technol., pp.253-261, 2001

[23] L. Jiang, S. Shiba, K. Shimbo, N. Sakai, T. Yamakura, M. Sugimura, P. G. Ananthasubramanian, H. Maezawa, Y. Irimajiri, and S. Yamamoto, "Development of THz waveguide NbTiN HEB mixers," IEEE Trans. Appl. Supercond., vol.19, no.3, pp.301-304, June 2009 .

[24] L. Jiang, S. Shiba, T. Shiino, K. Shimbo, N. Sakai, T. Yamakura, Y. Irimajiri, P. G. Ananthasubramanian, H. Maezawa, and S. Yamamoto, "Development of $1.5 \mathrm{THz}$ waveguide NbTiN superconducting hot electron bolometer mixers," Supercon. Sci. Technol., vol.23, no.4, pp.045025, Apr. 2010.

[25] H. Maezawa, T. Yamakura, T. Shiino, S. Yamamoto, S. Shiba, N. Sakai, Y. Irimajiri, L. Jiang, N. Nakai, M. Seta, A. Mizuno, T. Nagahama, and Y. Fukui, "Stability of a quasi-optical superconducting NbTiN hot-electron bolometer mixer at $1.5 \mathrm{THz}$ frequency band," IEEE Trans. Appl. Supercond., vol.21, no.3, pp.640-644, June 2011.

[26] T. Shiino, S. Shiba, N. Sakai, T. Yamakura, L. Jiang, Y. Uzawa, H. Maezawa, and S. Yamamoto, "Improvement of the critical temperature of superconducting NbTiN and $\mathrm{NbN}$ thin films using the AlN buffer layer," Supercon. Sci. Technol., vol.23, no.4, pp.045004, Apr. 2010.

[27] H. Maezawa, T. Sato, and T. Noguchi, "Process simulation of reac- 
tive DC magnetron sputtering for thin film deposition of niobiumtitanium nitride," IEEE Trans. Appl. Supercond., vol.15, no.2, pp.3520-3523, June 2005.

[28] J. Zmuidzinas and H. G. LeDuc, "Quasi-optical slot antenna SIS mixers," IEEE Trans. Microw. Theory Tech., vol.40, no.9, pp.17971804, Sept. 1992.

[29] P. Focardi, W. R. McGrath, and A. Neto, "Design guidelines for terahertz mixers and detectors," IEEE Trans. Microw. Theory Tech., vol.53, no.5, pp.1653-1661, May 2005.

[30] http://www.ticra.com/products/software/grasp

[31] P. Pütz, C. E. Honingh, K. Jacobs, M. Justen, M. Schultz, and J. Stutzki, "Terahertz hot electron bolometer waveguide mixers for GREAT," Astron. Astrophys., vol.542, pp.L2, June 2012.

[32] F. M. Boussaha, J. H. Kawamura, J. A. Stern, A. Skalare, and V. White, "A low noise $2.7 \mathrm{THz}$ waveguide-based superconducting mixer," IEEE Trans. Terahertz Sci. Technol., vol.2, no.3, pp.284289, May 2012.

[33] D. Meledin, A. Pavolotsky, V. Desmaris, I. Lapkin, C. Risacher, V. Perez, D. Henke, O. Nystrom, E. Sundin, D. Dochev, M. Pantaleev, M. Fredrixon, M. Strandberg, B. Voronov, G. Goltsman, and V. Belitsky, "A 1.3-THz balanced waveguide HEB mixer for the APEX telescope," IEEE Trans. Microw. Theory Tech., vol.57, no.1, pp.8998, Dec. 2009

[34] D. Dochev, V. Desmaris, D. Meledin, A. Pavolotsky, V. Belitsky, "A technology demonstrator for 1.6-2.0 THz waveguide HEB receiver with a novel mixer layout," J. Infrared., Milli. Terahz. Waves., vol.32, pp.451-465, Apr. 2011.

[35] A. Shurakov, C. E. Tong, R. Blundell, N. Kaurova, B. Voronov, and G. Gol'tsman, "Microwave stabilization of a HEB mixer in a pulse-tube cryocooler," IEEE Trans. Appl. Supercond., vol.23, no.3, pp.1501504, Jan. 2013.

[36] J. Kawamura, T. R. Hunter, C.-Y. E. Tong, R. Blundell, D. C. Papa, F. Patt, W. Peters, T. L. Wilson, C. Henkel, G. Gol'tsman, and E. Gershenzon, "Ground-based terahertz CO spectroscopy towards Orion," Astron. Astrophys., vol.394, no.1, pp.271-274, Oct. 2002.

[37] D. P. Marrone, R. Blundell, E. Tong, S. N. Paine, D. Loudkov, and J. H. Kawamura, "Observations in the 1.3 and $1.5 \mathrm{THz}$ atmospheric windows with the receiver lab telescope," 16th Int, Symp. Space Terahertz Technol., pp.64-67, 2005.

[38] D. P. Marrone, J. Battat, F. Bensch, R. Blundell, M. Diaz, H. Gibson, T. Hunter, D. Meledin, S. Paine, D. C. Papa, S. Radford, M. Smith, and E. Tong, "A Map of OMC-1 in CO $J=9 \rightarrow 8$," Astrophys. J., vol.612, no.2, pp.940-945, Sept. 2004.

[39] Th. de Graauw, et al. "The Herschel-Heterodyne Instrument for the Far-Infrared (HIFI)," Astron. Astrophys., vol.518, pp.L6, July-Aug. 2010

[40] G. L. Pilbratt, J. R. Riedinger, T. Passvogel, G. Crone, D. Doyle, U. Gageur, A. M. Heras, C. Jewell, L. Metcalfe, S. Ott, and M. Schmidt, "Herschel space observatory," Astron. Astrophys., vol.518, pp.L1, July-Aug. 2010.

[41] N. R. Crockett, E. A. Bergin, J. L. Neill, C. Favre, P. Schilke, D. C. Lis, T. A. Bell, G. Blake, J. Cernicharo, M. Emprechtinger, G. B. Esplugues, H. Gupta, M. Kleshcheva, S. Lord, N. Marcelino, B. A. McGuire, J. Pearson, T. G. Phillips, R. Plume, F. van der Tak, B. Tercero, and S. Yu, "Herschel observations of extraordinary sources: Analysis of the HIFI $1.2 \mathrm{THz}$ wide spectral survey toward Orion KL. I. methods, ” Astrophys. J., vol.787, no.2, pp.112, June 2014.

[42] T. Velusamy, W. D. Langer, J. L. Pineda, and P. F. Goldsmith, "[C II] $158 \mu \mathrm{m}$ line detection of the warm ionized medium in the ScutumCrux spiral arm tangency," Astron. Astrophys., vol.541, pp.L10, May 2012.

[43] P. Temi, P. M. Marcum, E. Young, J. D. Adams, S. Adams, B.G. Andersson, E. E. Becklin, A. Boogert, R. Brewster, E. Burgh, B. R. Cobleigh, S. Culp, J. De Buizer, E. W. Dunham, C. Engfer, G. Ediss, M. Füjieh, R. Grashuis, M. Gross, E. Harmon, A. Helton, D. Hoffman, J. Homan, M. Hutwohl, H. Jakob, S. C. Jensen, C. Kaminski, D. Kozarsky, A. Krabbe, R. Klein, Y. Lammen, U.
Lampater, W. B. Latter, J. Le, N. McKown, R. Melchiorri, A. W. Meyer, J. Miles, W. E. Miller, S. Miller, E. Moore, D. J. Nickison, K. Opshaug, E. Pfüeller, J. Radomski, J. Rasmussen, W. Reach, A. Reinacher, T. L. Roellig, G. Sandell, R. Sankrit, M. L. Savage, S. Shenoy, J. E. Schonfeld, R. Y. Shuping, E. C. Smith, E. Talebi, S. Teufel, T. C. Tseng, W. D. Vacca, J. Vaillancourt, J. E. Van Cleve, M. Wiedemann, J. Wolf, E. Zavala, O. Zeile, P. T. Zell, and H. Zinnecker, "The SOFIA observatory at the start of routine science operations: Mission capabilities and performance," Astrophys. J. Suppl., vol.212, no.2, pp.24, June 2014.

[44] H. Zinnecker, "SOFIA: First science highlights and future science potential," Astron. Nachr., vol.334, no.6, pp.558-575, July 2013.

[45] S. Heyminck, U. U. Graf, R. Güsten, J. Stutzki, H. W. Hübers, and P. Hartogh, "GREAT: The SOFIA high-frequency heterodyne instrument," Astron. Astrophys., vol.542, pp.L1, June 2012.

[46] G. Villanueva and P. Hartogh, "The high resolution chirp transform spectrometer for the Sofia-great instrument," Exp. Astron., vol.18, no.1-3, pp.77-91, Dec. 2004.

[47] H. Wiesemeyer, R. Güsten, S. Heyminck, K. Jacobs, K. M. Menten, D. A. Neufeld, M. A. Requena-Torres, and J. Stutzki, "Highresolution absorption spectroscopy of the $\mathrm{OH}^{2} \Pi_{3 / 2}$ ground state line," Astron. Astrophys., vol.542, pp.L7, June 2012.

[48] F. Wyrowski, R. Güsten, K. M. Menten, H. Wiesemeyer, and B. Klein, "Terahertz ammonia absorption as a probe of infall in highmass star forming clumps," Astron. Astrophys., vol.542, pp.L15, June 2012.

[49] B. Parise, F. Du, F.-C. Liu, A. Belloche, H. Wiesemeyer, R. Güsten, K. M. Menten, H.-W. Hübers, and B. Klein, "Detection of OD towards the low-mass protostar IRAS 16293-2422," Astron. Astrophys., vol.542, pp.L5, June 2012.

[50] D. A. Neufeld, E. Falgarone, M. Gerin, B. Godard, E. Herbst, G. Pineau des Forêts, A. I. Vasyunin, R. Güsten, H. Wiesemeyer, and O. Ricken, "Discovery of interstellar mercapto radicals $(\mathrm{SH})$ with the GREAT instrument on SOFIA," Astron. Astrophys., vol.542, pp.L6, June 2012

[51] R. Sahai, M. R. Morris, M. W. Werner, R. Gusten, H. Wiesemeyer, and G. Sandell, "Probing the mass and structure of the Ring Nebula in Lyra with SOFIA/GREAT observations of the [CII] 158 micron line," Astron. Astrophys., vol.542, pp.L20, 2012.

[52] M. C. Wiedner, G. Wieching, F. Bielau, K. Rettenbacher, N. H. Volgenau, M. Emprechtinger, U. U. Graf, C. E. Honingh, K. Jacobs, B. Vowinkel, K. M. Menten, L.-Å. Nyman, R. Güsten, S. Philipp, D. Rabanus, J. Stutzki, and F. Wyrowski, "First observations with CONDOR, a $1.5 \mathrm{THz}$ heterodyne receiver," Astron. Astrophys., vol.454, no.2, pp.L33-L36, Aug. 2006.

[53] A. D. Semenov, H. Richter, H.-W. Hübers, P. Khosropanah, M. Hajenius, J.-R. Gao, and T. M. Klapwijk, "Development of a 1.8-THz hot-electron-bolometer mixer for TELIS," 19th Int. Symp. Space Terahertz Technol., pp.417-420, 2008.

[54] H.-W. Hübers, R. Eichholz, S. G. Pavlov, and H. Richter, "High resolution terahertz spectroscopy with quantum cascade lasers," J. Infrared, Millimeter, Terahertz Waves, vol.34, no.5-6, pp.325-341, June 2013.

[55] B. S. Williams, "Terahertz quantum-cascade lasers," Nat. Photonics, vol.1, no.9, pp.517-525, Sept. 2007.

[56] S. Kumar, B. S. Williams, S. Kohen, Q. Hu, and J. L. Reno, "Continuous-wave operation of terahertz quantum-cascade lasers above liquid-nitrogen temperature," Appl. Phys. Lett., vol.84, no.14, pp.2494-2496, Apr. 2004.

[57] J. R. Gao, J. N. Hovenier, Z. Q. Yang, J. J. A. Baselmans, A. Baryshev, M. Hajenius, T. M. Klapwijk, A. J. L. Adam, T. O. Klaassen, B. S. Williams, S. Kumar, Q. Hu, and J. L. Reno, "Terahertz heterodyne receiver based on a quantum cascade laser and a superconducting bolometer," Appl. Phys. Lett., vol.86, no.24, pp.244104, June 2005.

[58] S. Shiba, Y. Irimajiri, T. Yamakura, H. Maezawa, N. Sekine, I. Hosako, and S. Yamamoto, "3.1-THz heterodyne receiver using an 
NbTiN hot-electron bolometer mixer and a quantum cascade laser," IEEE Trans. Terahertz Sci. Technol., vol.2, no.1, pp.22-28, Jan. 2012.

[59] Y. Ren, J. N. Hovenier, M. Cui, D. J. Hayton, J. R. Gao, T. M. Klapwijk, S. C. Shi, T.-Y. Kao, Q. Hu, and J. L. Reno, "Frequency locking of a $3.5 \mathrm{THz}$ quantum cascade laser using a gas cell," 22 nd Int. Symp. Space Terahertz Technol., pp.203, 2011.

[60] A. M. Baryshev, P. Khosropanah, W. Zhang, W. Jellema, J. N. Hovenier, J. R. Gao, T. M. Klapwijk, D. G. Paveliev, B. S. William, S. Kumar, Q. Hu, J. L. Reno, B. Klein, and J. L. Hesler, "Phase-locking of a 2.7-THz quantum cascade laser to a microwave reference," 20th Int, Symp. Space Terahertz Technol., pp.49, 2009.

[61] A. M. Baryshev, J. N. Hovenier, A. J. L. Adam, I. Kašalynas, J. R. Gao, T. O. Klaassen, B. S. Williams, S. Kumar, Q. Hu, and J. L. Reno, "Phase locking and spectral linewidth of a two-mode terahertz quantum cascade laser," Appl. Phys. Lett., vol.89, no.3, pp.031115, July 2006.

[62] D. Rabanus, U. U. Graf, M. Philipp, O. Ricken, J. Stutzki, B. Vowinkel, M. C. Wiedner, C. Walther, M. Fischer, and J. Faist, "Phase locking of a 1.5 Terahertz quantum cascade laser and use as a local oscillator in a heterodyne HEB receiver," Opt. Express, vol.17, no.3, pp.1159-1168, Feb. 2009.

[63] J. L. Kloosterman, D. J. Hayton, Y. Ren, T. Y. Kao, J. N. Hovenier, J. R. Gao, T. M. Klapwijk, Q. Hu, C. K. Walker, and J. L. Reno, "Hot electron bolometer heterodyne receiver with a 4.7-THz quantum cascade laser as a local oscillator," Appl. Phys. Lett., vol.102, no.1, pp.011123, Jan. 2013.

[64] Y. Ren, J. N. Hovenier, R. Higgins, J. R. Gao, T. M. Klapwijk, S. C. Shi, A. Bell, B. Klein, T.-Y. Kao, S. Kumar, Q. Hu, and J. L. Reno, "Gas cell measurement using a $2.9 \mathrm{THz}$ heterodyne receiver based on a quantum cascade laser and a superconducting hot electron bolometer," 21 st Int, Symp. Space Terahertz Technol., pp.229, 2010.

[65] Y. Ren, J. N. Hovenier, R. Higgins, J. R. Gao, T. M. Klapwijk, S. C. Shi, B. Klein, T.-Y. Kao, Q. Hu, and J. L. Reno, "High-resolution heterodyne spectroscopy using a tunable quantum cascade laser around 3.5 THz," Appl. Phys. Lett., vol.98, no.23, pp.231109, June 2011.

[66] M. I. Amanti, M. Fischer, G. Scalari, M. Beck, and J. Faist, "Lowdivergence single-mode terahertz quantum cascade laser," Nat. Photonics, vol.3, no.10, pp.586-590, Oct. 2009.

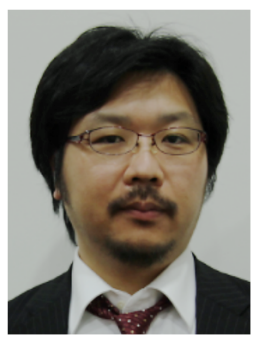

Hiroyuki Maezawa received his M.S. and Ph.D. degrees in physics from the University of Tokyo, Tokyo, Japan, in 1998, and 2001, respectively. He worked at the Nobeyama Radio Observatory (NRO) and the National Astronomical Observatory (NAO) from 2001 to 2003 to serve on the ALMA project. During 2004-2011, he was an Assistant Professor at Nagoya University. He is currently an Associate Professor in the Astrophysics Group of the Department of Physical Science, Osaka Prefecture University, and a guest fellow of the Department of Astrophysics, Nagoya University and Institute of Space and Astrophysical Science/Japan Aerospace Exploration Agency (ISAS/JAXA). His research interests include astrophysics, earth and planetary sciences, and terahertz-sensing technologies. 